\title{
New experimental techniques for fracture testing of highly deformable materials
}

\author{
E. Dall'Asta, V. Ghizzardi, R. Brighenti, E. Romeo, R. Roncella, A. Spagnoli \\ DICATeA, University of Parma, 43124 Parma (PR), Italy \\ dallastaelisa@gmail.com,valeria.ghizzardi@gmail.com,riccardo.roncella@unipr.it,roberto.brighenti@unipr.it, \\ andrea.spagnoli@unipr.it
}

\begin{abstract}
A new experimental method for measuring strain fields in highly deformable materials has been developed. This technique is based on an in-house developed Digital Image Correlation (DIC) system capable of accurately capturing localized or non-uniform strain distributions. Thanks to the implemented algorithm based on a Semi-Global Matching (SGM) approach, it is possible to constraint the regularity of the displacement field in order to significantly improve the reliability of the evaluated strains, especially in highly deformable materials. Being originally introduced for Digital Surface Modelling from stereo pairs, SGM is conceived for performing a one-dimensional search of displacements between images, but here a novel implementation for 2D displacement solution space is introduced. SGM approach is compared with the previously in-house developed implementation based on a local Least Squares Matching (LSM) approach. A comparison with the open source code Ncorr and with some FEM results is also presented. The investigation using the present DIC method focuses on 2D full-field strain maps of plain and notched specimens under tensile loading made of two different highly deformable materials: hot mix asphalt and thermoplastic composites for 3D-printing applications. In the latter specimens, an elliptical hole is introduced to assess the potentiality of the method in experimentally capturing high strain gradients in mixed-mode fracture situations.
\end{abstract}

KeYwords. Digital Image Correlation; Highly Deformable Materials; Mixed-mode fracture; Mastic; Polylactide.

\section{INTRODUCTION}

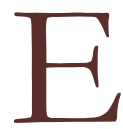
xperimental solid mechanics testing often requires accurate measurements of full-field strain components. The term Digital Image Correlation (DIC) refers to a class of non-contacting techniques that utilize two or more digital images depicting (at least partly) the same object and, performing image analysis, are capable of extracting a full field description of the shape, displacements and/or deformations of the object itself. Indeed, the improvement in image processing by means of microcomputers has fostered non-contact measurement techniques to become more and more popular in the experimental mechanics community [1], in comparison to some full-field measurement techniques like Moiré, interferometry or photoelasticimetry which were known and used beforehand but with a difficult trade-off between results accuracy, simplicity and costs. In the last decade, the opportunity to obtain displacement or strain contours directly (by automatic image processing), with low costs and high accuracy, has determined an increased use of optical devices, digital cameras, algorithms and software. DIC was originally proposed in the 1980's [2] to study 2D solid mechanics problems, such as resin films [3], fiber reinforced polymer composites [4] and concrete [5]. Kim and Wen [6] first proposed the use of DIC technique as a possible displacement/strain measurement method for asphalt mixture. Seo et al. [7] utilized a DIC technique to investigate the size and shape of the fracture process zone for asphalt mixtures. 
Masad et al. [8] used both digital imaging and X-ray computed tomography techniques to evaluate the microstructure of hot mix asphalts in terms of aggregate orientation and air voids concentration, as well as strain distribution. Birgisson et al. [9] used DIC to validate the theory at the base of the visco-elastic fracture mechanics-based crack growth, which identifies a fundamental crack growth threshold as the key element in defining the cracking mechanism and fracture resistance of asphalt mixtures. Nowadays, DIC strain measurement is a continuously improving technique: for instance, Nashon et. al. [10] have analysed the ductile fracture of aluminum panels, using the obtained results also as calibration and validation data for the numerical modeling of ductile fracture in large structures. Strain and displacement analysis of specific and unusual materials were recently tested by using DIC: Makki et. al. [11] have presented the stress localization and concentration for isotropic and orthotropic materials with holes and, in Petrikova et. al. [12], the big deformations of a hyperelastic material are investigated. Recent advances and perspectives in DIC and related methods for accurate, full-field deformation mappings have finally been described [13].

In this paper, 2D full-field strain experimental measurements of plain and notched specimens under tensile loading, using DIC technique, are performed. Two materials with different areas of application, both characterized by a high deformability, are investigated: hot mix asphalt for road pavements and thermoplastic composites for 3D-printing. The final goal is to verify the applicability of DIC technique in the measurement of large displacements and high deformations in the presence of strain gradients. On one hand, non-homogeneous strain fields are explored in the case of hot mix asphalt mixture by studying mastic elements, composed by binder and filler. On the other hand, notched specimens of thermoplastic polymers with elliptical holes are considered in order to investigate mixed-mode fracture initiation of the material. Strain localization and damage distribution are measured using both an open source Matlab code Ncorr [14] and a proprietary DIC software developed at the University of Parma. Thanks to the in-house implementation of two different image-matching algorithms, the oldest, based on Least Squares Matching (LSM) and the newest, based on Semi-Global Matching (SGM) techniques, the reliability of the measured deformation, especially in highly deformable materials, can be assessed. The latter technique is based on a recent stereo matching method [15] that is rapidly spreading in Photogrammetry and Computer Vision communities. Until now it has been successfully applied for 1D displacement field estimation (for example for stereo vision applications) and never tested in DIC application where the full 2D displacement field of the specimen is commonly required. For this reason, a novel implementation of the SGM technique with an algorithmic extension to a $2 \mathrm{D}$ displacement search domain has been developed and tested.

This paper summarizes the most significant experimental studies performed using DIC-based techniques on the two different high deformable materials, comparing strains registered by different DIC stereo matching algorithms with finite element simulations.

\section{THEORETICAL PRINCIPLES OF DIC}

$\mathrm{D}$ IC is a non-contact optical technique which allows measurement of displacements and strains in engineering materials. It works by tracking the same points between two consecutive images (before and under loading) of the material specimen at different stage of its deformation. The feature tracking is usually achieved using Area Based Matching $(\mathrm{ABM})$, a technique for the extraction of image correspondences based on similarities between grey values. In ABM, each image point to be matched is the centre of a small pixels window (template) in a selected reference image (master image), which usually corresponds to the first image (before loading) in the sequence of frames. The grey values of the template are compared with those of an equally sized pixels (patch) in a deformed search image (slave image).

In short, all the different image matching techniques aim at the same result: comparing images that present radiometric and geometric differences due to a relative (three-dimensional) motion between the camera (the observer) and the object (the scene framed by the camera), and tracking the (two-dimensional) movement of specific elements on the image. DIC performances depend on the algorithm capability to identify the same feature in different images: well-contrasted and recognizable pattern facilitate the tracking process, achieving good accuracies and high matching reliability.

To draw a boundary line between the plethora of techniques that can be used for image matching applications, a first criterion is the kind of image elements that are tackled by the algorithm. Accordingly, we can distinguished between: (i) Local methods (e.g. Least Squares Matching) which track the position of one point at a time comparing the area (i.e. the image block) surrounding the point itself (neighbouring points and thus neighbouring measures are not influenced by each other); (ii) Global/semi-global methods which estimate the whole displacement field of the Region Of Interest, ROI (in many cases the ROI corresponds to the full image extent) in a single pass (every point affects the neighbouring ones and 
the problem of finding the correspondences deals with the minimization of a global cost function extended usually to all image pixels).

\section{Least Squares Matching}

Least Square Matching (LSM) method consists in the minimization of the squared differences of the grey values between patch and template. LSM uses a functional model, which is able to provide matches with sub pixel accuracy while accounting for some deformation of the patch block with respect to the template window. In this approach, the matching process consists in the estimation of the function parameters that maximize the similarity between the template and the (deformed) patch, using a least squares solution [16]. Given two image points, the least squares matching considers the two conjugate image regions as discrete two-dimensional functions: the template $\mathrm{f}\left(\mathrm{x}_{1}, \mathrm{y}_{1}\right)$ and the patch $\mathrm{g}\left(\mathrm{x}_{2}, \mathrm{y}_{2}\right)$. The patch is transformed applying both radiometric and geometric adjustments to obtain a new patch $\mathrm{g}^{\prime}\left(\mathrm{x}_{2}, \mathrm{y}_{2}\right)$. The matching process establishes a correspondence minimizing the $\mathrm{L} 2$-norm of the residual vector $\mathrm{e}\left(\mathrm{x}_{1}, \mathrm{y}_{1}\right)$ :

$$
f\left(x_{1}, y_{1}\right)-g^{\prime}\left(x_{2}, y_{2}\right)=e\left(x_{1}, y_{1}\right)
$$

Radiometric changes (due to contrast and brightness variations of intensity values in the slave image) are modelled in the patch function as:

$$
\mathrm{g}^{\prime}\left(\mathrm{x}_{2}, \mathrm{y}_{2}\right)=\mathrm{r}_{0}+\left(1+\mathrm{r}_{1}\right) \cdot \mathrm{g}\left(\mathrm{x}_{2}, \mathrm{y}_{2}\right)
$$

where $r_{0}$ and $r_{1}$ are two parameters accounting for brightness and contrast changes in the slave image, respectively. Geometric corrections are considered by means of a geometrical parametric transformation:

$$
\mathrm{g}\left(\mathrm{x}_{2}, \mathrm{y}_{2}\right)=\mathrm{g}\left(\mathrm{x}_{2}\left(\mathrm{x}_{1}, \mathrm{y}_{1}\right), \mathrm{y}_{2}\left(\mathrm{x}_{1}, \mathrm{y}_{1}\right)\right)
$$

The following affine transformation model is commonly used in DIC applications:

$$
\left\{\begin{array}{l}
x_{2}=a_{1} x_{1}+a_{2} y_{1}+a_{3} \\
y_{2}=b_{1} x_{1}+b_{2} y_{1}+b_{3}
\end{array}\right.
$$

where $\left(a_{1}, a_{2}, b_{1}, b_{2}\right)$ model shape differences between patch and template, while $\left(a_{3}, b_{3}\right)$ are the shift parameters. Such transformation is considered as the optimal choice for taking into account the large anisotropic deformation of the slave image and for limiting, at the same time, the numerical complexity (and consequently instability) of the geometric model. While more simplified shape function models becomes rapidly not affordable to identify the exact localization of the template centre, even with isotropic scale deformations, higher order transformations require more observation to be appropriately estimated (and consequently a wider template area which can present strong localized deformation especially with composite high deformable materials). The very same behaviour is shown quantitatively in [17], even if in that case the matching algorithm is applied to planetary surface reconstruction.

Radiometric and geometric correction parameters are then estimated solving, for $\|\mathrm{e}(\mathrm{x} 1, \mathrm{y} 1)\|=\min$, the following least squares system, obtained by substituting the transformed functions in Eq. 1:

$$
\mathrm{f}\left(\mathrm{x}_{1}, \mathrm{y}_{1}\right)+\mathrm{e}\left(\mathrm{x}_{1}, \mathrm{y}_{1}\right)=\mathrm{r}_{0}+\mathrm{r}_{1} \cdot \mathrm{g}\left(\mathrm{x}_{2}\left(\mathrm{x}_{1}, \mathrm{y}_{1}\right), \mathrm{y}_{2}\left(\mathrm{x}_{1}, \mathrm{y}_{1}\right)\right)=\bar{g}\left(\mathrm{r}_{0}, \mathrm{r}_{1} \mathrm{a}_{1}, \mathrm{a}_{2} \mathrm{a}_{3}, \mathrm{~b}_{1}, \mathrm{~b}_{2}, \mathrm{~b}_{3}\right)
$$

The function $\overline{\mathrm{g}}\left(\mathrm{r}_{0}, \mathrm{r}_{1}, \mathrm{a}_{1}, \mathrm{a}_{2}, \mathrm{a}_{3}, \mathrm{~b}_{1}, \mathrm{~b}_{2}, \mathrm{~b}_{3}\right)$ is linearized and the system is commonly solved by Gauss-Markov least squares estimation model.

\section{Semi-Global Matching}

The Semi-Global Matching method [15] performs a pixel-wise matching, considering both the image similarity and the displacement continuity, by the concurrent application of regularization constraints (in terms of adiacent pixels displacement). It realizes the minimization of a global cost function, combining matching costs along indipendent onedimensional paths from all directions through the image. The costs extracted by each path, refered to a particular displacement value, are summed for each pixel and possible displacement (also referred as disparity) value. Finally, the 
algorithm choses the disparity solution with the minimum cost, using algorithms like Dynamic Programming (DP) [18], Graph Cuts [19] or Belief Propagation [20]. In a DP framework, as the ones implemented in many SGM software packages [21] the cost $\mathrm{L}_{\mathbf{r}}^{\prime}(\mathbf{p}, \mathrm{d})$ of the pixel located in $\mathbf{p}$ at disparity $\mathrm{d}$, along the path direction $\mathbf{r}$ is defined recursively as:

$$
\mathrm{L}_{\mathbf{r}}^{\prime}(\mathbf{p}, \mathrm{d})=\mathrm{C}(\mathbf{p}, \mathrm{d})+\min _{\mathrm{i}}\left\{\mathrm{L}_{\mathbf{r}}\left(\mathbf{p}-\mathbf{r}, \mathrm{d}+\Delta \mathrm{d}_{\mathrm{i}}\right)+\mathrm{P}\left(\Delta \mathrm{d}_{\mathrm{i}}\right)\right\}
$$

where the first term is the similarity cost associated with a disparity value of $\mathrm{d}$, whereas the second term evaluates the regularity of the disparity field, adding a penalty term $\mathrm{P}$, function of disparity changes $\left(\Delta \mathrm{d}_{\mathrm{i}}\right)$ with respect to the previous point in the considered matching path $\mathbf{r}$.

The final aggregated cost, that takes into account all the different $\boldsymbol{r}$ paths, is defined as

$$
\mathrm{S}(\mathrm{p}, \mathrm{d})=\sum_{\mathrm{r}} \mathrm{L}_{\mathrm{r}}(\mathrm{p}, \mathrm{d})
$$

and, for sub-pixel estimation of the final disparity solution, the position of the minimum is usually calculated by fitting a quadratic curve through the cost values of neighboring pixels.

Indeed, these methods successfully combine concepts of global and local stereo methods for accurate, pixel-wise matching at low runtime and, consequently, works particularly well for 1D displacement (or disparity) field calculation. In many applications, e.g. in stereo-vision problems, the images to be matched can be transformed so that the displacement between conjugated corresponding points occurs always along the same direction (e.g. see rectified images in [22]).

In DIC applications (as well as other applications like Particle Image Velocimetry, PIV [23]) the displacements of conjugated points are actually two-dimensional and traditional SGM algorithms cannot be used. In particular eq. (8) should be extended to consider the 2D search domain:

$$
\mathrm{L}_{\mathbf{r}}^{\prime}(\mathbf{p}, \mathrm{d})=\mathrm{C}(\mathbf{p}, \mathrm{dx}, \mathrm{dy})+\min _{\mathrm{i}, \mathrm{j}}\left\{\mathrm{L}_{\mathbf{r}}\left(\mathbf{p}-\mathbf{r}, \mathrm{dx}+\Delta \mathrm{d}_{\mathrm{i}}, \mathrm{dy}+\Delta \mathrm{d}_{\mathrm{i}}\right)+\mathrm{P}\left(\Delta \mathrm{d}_{\mathrm{i}}, \Delta \mathrm{d}_{\mathrm{i}}\right)\right\}
$$

Where $\mathrm{dx}$ and $\mathrm{dy}$, and $\Delta \mathrm{d}_{\mathrm{i}}$ and $\Delta \mathrm{d}_{\mathrm{j}}$, are respectively the displacement components and the disparity changes (as defined for eq. (7)) along the principal image plane axis.

The high amount of memory and calculations required by the original SGM further increases introducing the 2D disparity search option. It is easy to note that a $1 \mathrm{D}$ disparity search domain makes the complexity of the problem proportional to $\mathrm{O}(\mathrm{m} \cdot \mathrm{n} \cdot \mathrm{d})$, where $\mathrm{m}$ and $\mathrm{n}$ are the pixel resolution of the image and $\mathrm{d}$ is the disparity search range, while, in the novel approach, the complexity tends to $\mathrm{O}\left(\mathrm{m} \cdot \mathrm{n} \cdot \mathrm{d}^{2}\right)$. For this reason, a multi-resolution scheme is implemented to limit the disparity search range as much as possible. At the same time, being the total disparity range usually very wide, especially with highly deformable materials, a data structure that saves the expected central displacement value of each pixel is used to further limit the disparity range.

The introduction of regularity constraints allows the use of very small templates (usually 1 to 5 pixels) making the method particularly robust where shape discontinuities are present as well as when high deformations occur. Even with a very simple cost function for the similarity term $\mathrm{C}(\mathrm{p}, \mathrm{dx}, \mathrm{dy})$, e.g. the Sum of Absolute Difference (SAD) [24], where no patch deformation are considered (i.e. the patch can only translate), the method can produce good results, at least if image deformation in less than 30-35\%. In all these cases the most important tuning is represented by the penalty term $\mathrm{P}$ which involves the correct identification and application of a parametric regularization function applied to displacement differences of adjacent pixels.

\section{General precaution in DIC application to highly deformable materials}

Both the previously described image matching techniques can successfully applied to highly deformable materials. Nonetheless some precaution should be provided to ensure that the final results is as accurate and affordable as possible. First of all, sample preparation must be designed so that every area of the specimen is uniformly contrasted and no monochromatic area are present, since such areas, at high deformation rates, can become much bigger and can produce unpredictable results, regardless of the matching algorithm used. 
If the maximum deformation to be measured is less than $30 \%$ the use of simple similarity cost function (like SAD) can be used without any trouble with SGM algorithms. However, with these approaches and as far as the displacements are limited, pixel-locking [25] effects, which sometimes occur during the parabolic sub-pixel fitting of the cost function minimization, can produce unwanted biased results, as is evident in some of the results obtained below). Since the $2 \mathrm{D}$ displacement search domain should be very limited to improve the computational time required for the analysis, the differences between consecutive frames must be as small as possible. Namely, the frame rate during the test should be small enough to ensure that the maximum displacement between consecutive frame in image space is limited to some $(1 \div 5)$ pixels.

If higher deformation rates are expected, SGM algorithms should be discarded: the use of more complex similarity functions that consider also a patch deformation model, even if in principle can be implemented, will make the entire process computational unfeasible. At the same time, the simplistic regularization function in (8) can be unsuitable to constrain the displacement field of the solution which, with high deformation, can show localized effects more likely. Providing appropriate regularization functions and penalization terms can be tricky and introduce biases in the final results. In this context we found that the use of other cost function (i.e. Census transform cost function [24]) improves to some extent the results.

Anyway, in all these cases the use of LSM-like algorithms should be preferred: even with simple affine patch deformation model the accuracy obtainable is very good. The use of not too big templates (13 to 21 pixels) can provide affordable results, capturing localized effects with good computational times.

A final remark concerns the possibility to help the matching algorithm through the change of the master (reference) image during the DIC processing: in this way, comparing more similar images should improve the matching algorithm performance (no matter if LSM or SGM is used). Even if, in principle, that is true, the user have to consider that, in this way, accumulating the displacements computed from every reference and slave image, also the errors accumulate; in the end, it is likely that the entire process becomes less accurate than the case where the master image is fixed.

\section{MATERIALS AND SPECIMENS}

Mastic

sphalt mixture is a particulate composite material consisting of interspersed aggregates, asphalt binder and air voids. Its cracking behaviour is affected by its heterogeneity, specifically by the interaction between the aggregates and the mastic. The stiffness of the mastics affects the ability of the mixture to resist permanent deformation and their fracture and fatigue strength. Accurate description of strain evolution and distribution in mastics is essential for revealing significant information on the influence of microstructure properties on asphalt mixture macroscopic behaviour. Eight asphalt mastics with very different properties were used in this study. The cracking behaviour of the mastics is investigated using a Modified Direct Tension Test (MDTT) performed in a servo-hydraulic load frame to allow the material to stretch up to rupture. The specimen geometry, previously optimized to obtain uniform stress distribution within the specimen web and adequate gauge length for accurate strain measurements, is $106 \mathrm{~mm}$ long, $20 \mathrm{~mm}$ wide and $10 \mathrm{~mm}$ thick, with an effective gauge length of $46 \mathrm{~mm}$. Stroke applied is $1.68 \mathrm{~mm} / \mathrm{s}$ until rupture occurs.

A digital Basler piA1600-35gm camera (resolution 1608x1308, focal length 8mm, pixel size 7.4 micrometers, 35 fps@max resolution), directly connected to the testing control system, is located on a support inside the climatic chamber where tests are performed. The chamber is provided with a proper LED lighting system which assures good illumination without heating up the specimen. Since the crack phenomenon is very fast and short-lasting (1-2 seconds), the camera is properly set up to acquire the images in a smaller area of the sensor $(1600 \times 500$ pixel) reducing the bandwidth required for transmitting each frame and, consequently, allowing a higher frame rate (about $80 \mathrm{fps}$ ). Thanks to the elongated shape of the specimen, once provided an optimal imaging geometry, the reduced size of the images still allows the complete acquisition of the whole specimen surface. The images are automatically processed by the software, providing accurate displacement/strain fields. To achieve high accuracies in the strain field measurements, the specimen surface must present a well-contrasted grey scale speckle pattern, easily obtainable by a water paint-based treatment.

Strains are obtained from DIC system, interpolating all the strain values of the grid points located at the $46 \times 20 \mathrm{~mm}$ specimen central area. An example of the test configuration at failure is shown in Fig. 1(a).

\section{Plastic materials}

Polylactic acid or polylactide (PLA) is a biodegradable thermoplastic aliphatic polyester derived from renewable resources. PLA is commonly processed by 3D printing. In this work, PLA specimens are produced by a FFF (Fused Filament 
Fabrication) 3D printing technique, which is based on a layer-by-layer deposition, controlled by a slicing software, of plastic filaments (of 0.3 to $0.5 \mathrm{~mm}$ in diameter) supplied by an extrusion nozzle. PLA can be mechanically characterized by a visco-elastic behaviour which can be described for instance by the Maxwell-Wiechert model.

Tensile tests are performed on five 3D printed rectangular specimens with width $80 \mathrm{~mm}$, length $162 \mathrm{~mm}$ (clear length $119.2 \mathrm{~mm}$ ) and thickness $2 \mathrm{~mm}$, containing a central hole with maximum size of $30 \mathrm{~mm}$ and different shape and orientation: (i) a circular hole; (ii) an elliptical hole with aspect ratio 0.2 normal to the load axis; (iii) an elliptical hole, with aspect ratio 0.1 , normal to the load axis; (iv) an elliptical hole, with aspect ratio $0.1,60^{\circ}$ inclined with respect to the load axis; (v) an elliptical hole, with aspect ratio $0.1,45^{\circ}$ inclined with respect to the load axis. The load is acting in the direction of the major dimension of the specimens and is applied by displacement control of about $0.3 \mathrm{~mm}$ every 30 s. Note that in each layer the deposition of plastic filaments follows the $45^{\circ}$ direction with respect to the load axis, so as to induce some degree of orthotropy in the 3D printed specimens.

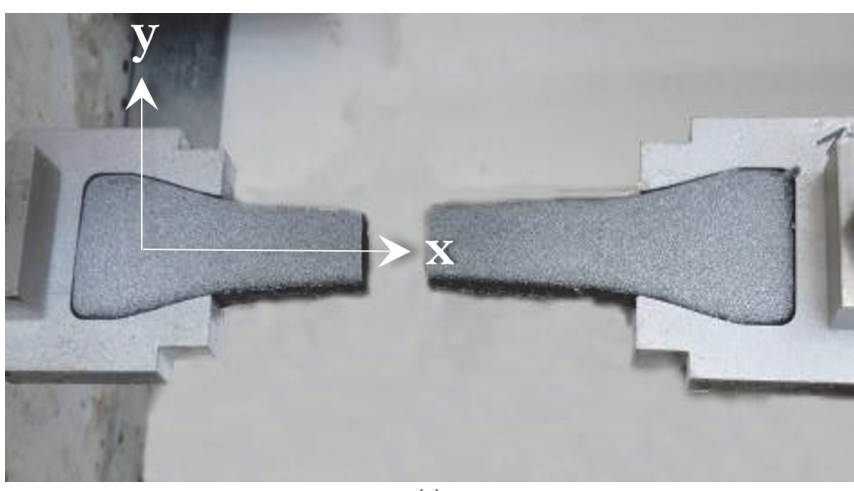

(a)

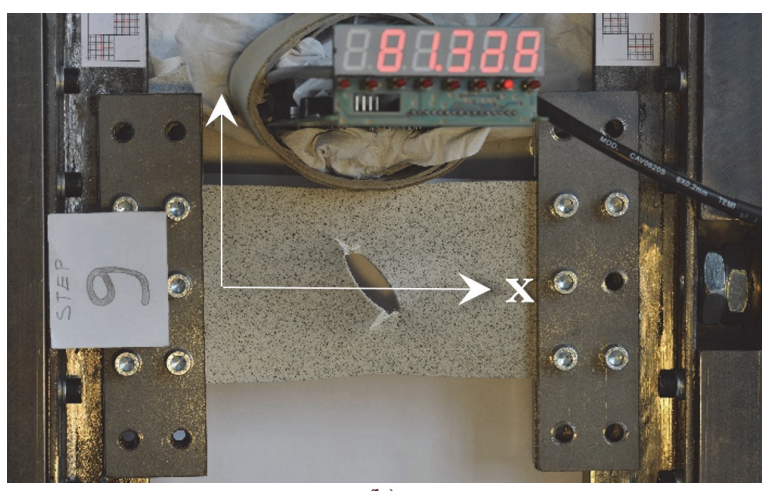

(b)

Figure 1: (a) Modified direct tension test for a mastic specimen at failure; (b) Tensile test for a polylactic specimen.

A full-format Nikon D3X (6048x4032 pixels) digital camera, with $105 \mathrm{~mm}$ lens and 6 micrometers pixel size, was used for DIC experiment. The camera was opportunely located on a tripod, in order to totally capture the sample, and the images were taken manually at defined temporal instants. Specimens have been treated before testing by applying an irregular painted surface pattern in order to get a nonuniform well-contrasted coloured surface suitable for performing DIC analyses. An example of the test configuration at failure is shown in Fig. 1(b).

\section{RESULTS}

$\mathrm{F}$ or comparison purposes, the in-house DIC system based on both LSM and SGM methods along with that of the open source code Ncorr are employed to obtain experimental measures of 2D full-field displacements and strains maps for mastic and PLA specimens. Deformation components are calculated according to the Green-Lagrange finite strain tensor, which, in the case of small displacement gradient compared to unity, coincides with the small strain tensor.

In the following, some selected contour maps of displacements and strains are reported, bearing in mind that $\mathrm{X}$ is aligned with the loading axis (see Fig. 1). The results correspond to an applied nominal strain equal to about 30\% of the strain at failure. Figs. 2-3 present the asphalt mastic specimens contour maps whereas, for PLA specimens, results for elliptical hole, with aspect ratio 0.1, normal to the load axis, and for elliptical hole, with aspect ratio $0.1,60^{\circ}$ inclined with respect to the load axis are reported in Figs. 4-6. The longitudinal nominal strain is equal to $2.01 \%$ and $3.36 \%$, respectively.

\section{COMPARISON WITH FE AND DISCUSSION}

$\mathrm{F}$ inite Element (FE) models of the PLA plates containing an elliptical hole are performed. Eight node finite elements in plane stress conditions are used. The FE model is loaded in tension by imposing a null axial displacement at one end and a uniform displacement at the opposite end. Linear and geometrically non-linear analyses are performed. 
The elastic equilibrium solution of the plane problem is related to prescribed boundary conditions, with no traction and no body forces being applied. The displacements/strains solution is independent on the Young modulus E (while stresses clearly depends on E), but it does depend on the Poisson ratio $v$ (a value of $v=0.45$ is adopted, which is typical of plastic materials and it is consistent with the experimental measurements of transversal contraction). This holds true also for the geometrically non-linear analyses with finite strains. The distribution of Green-Lagrange local strain components along the direction of the major axis of ellipse as a function of the distance from the ellipse root is compared in Fig. 7 for FE analysis and in-house DIC method using the LSM algorithm.
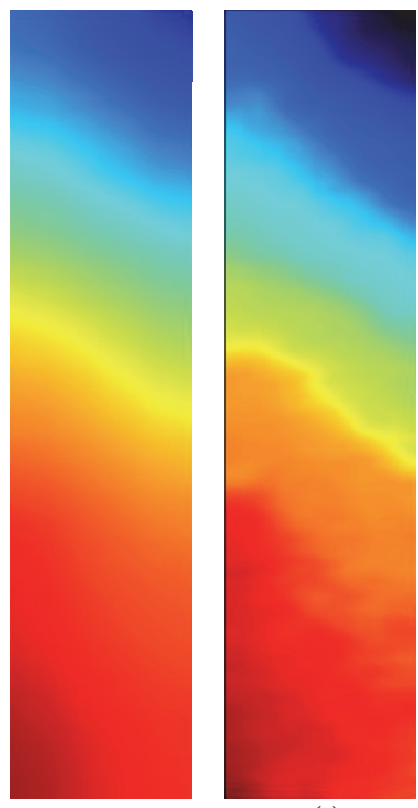

(a)

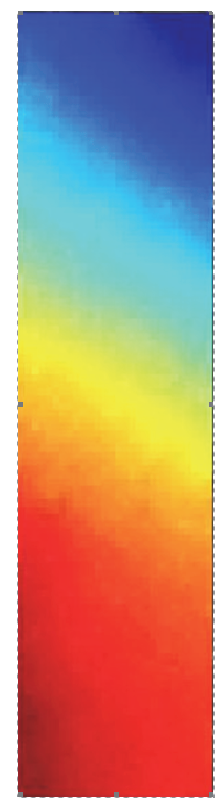

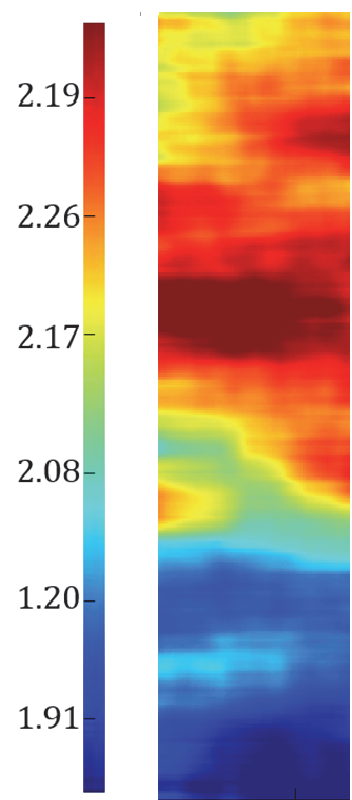
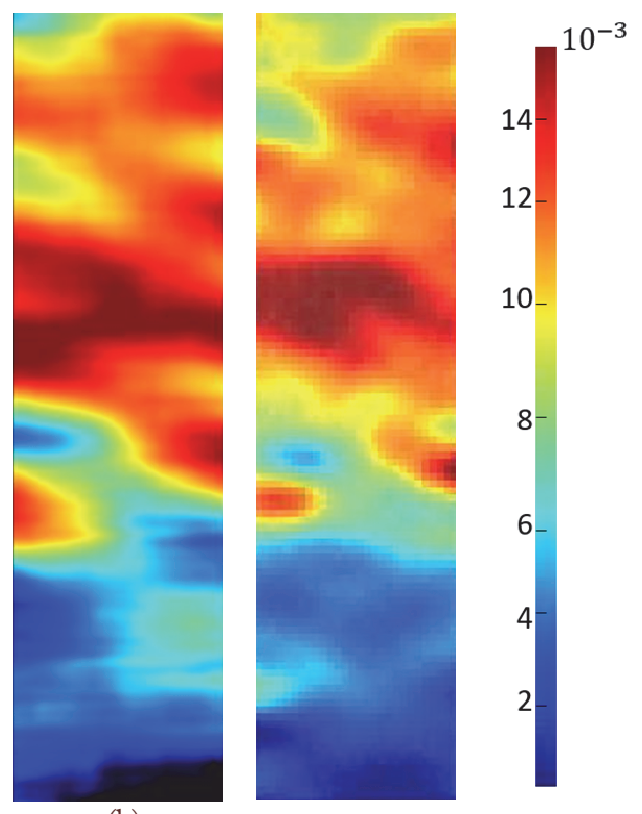

(b)

Figure 2: Asphalt mixture sample 1: results computed, in order of appearance, with LSM, SGM, NCORR. (a) Map of the displacement along $\mathrm{X}$ axis [mm]; (b) Map of the strain along $\mathrm{X}$ axis [-].
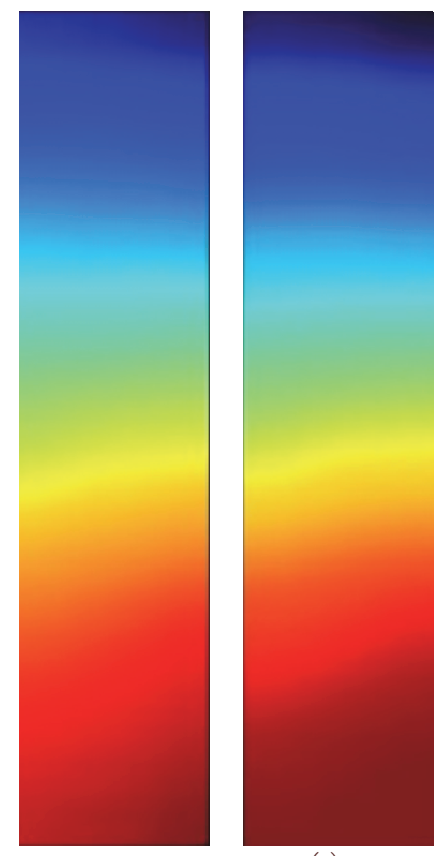

(a)

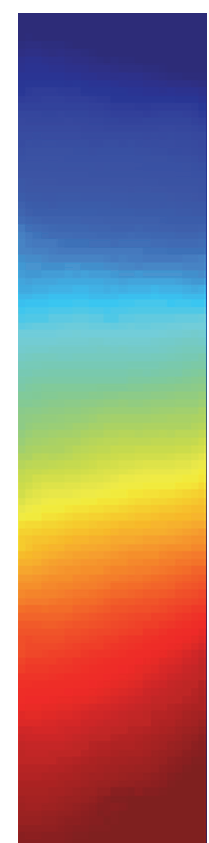

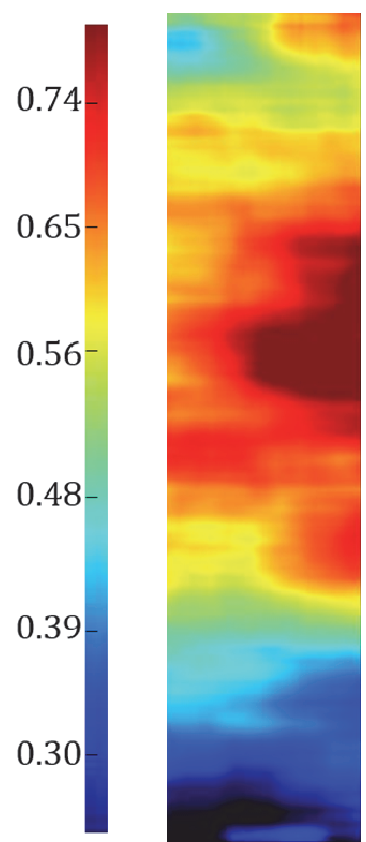
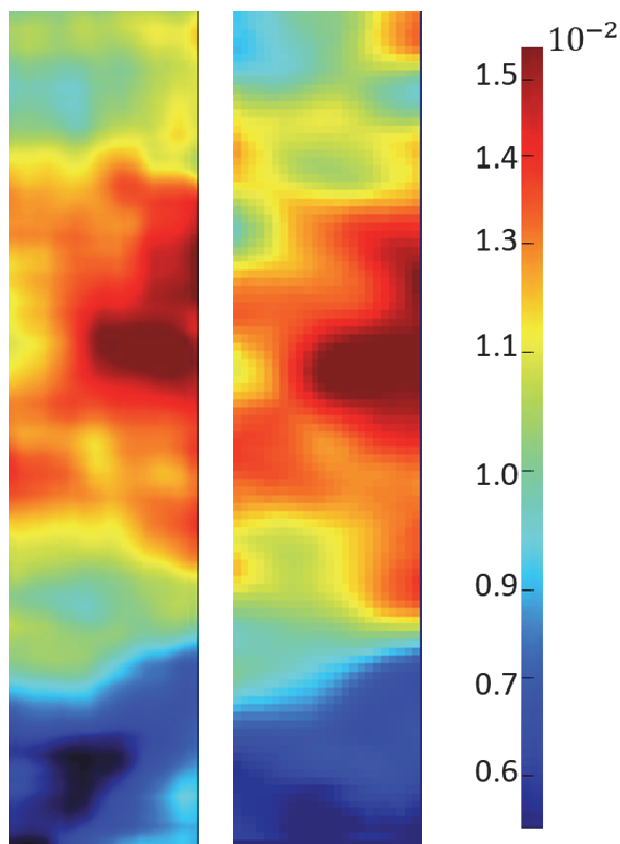

(b)

Figure 3: Asphalt mixture sample 2: results computed, in order of appearance, with LSM, SGM, NCORR. (a) Map of the displacement along $\mathrm{X}$ axis [mm]; (b) Map of the strain along $\mathrm{X}$ axis [-]. 


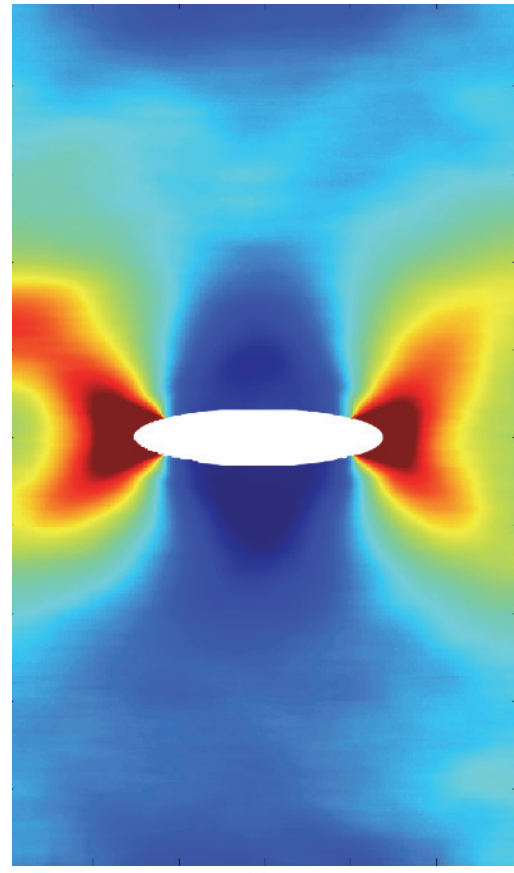

(a)

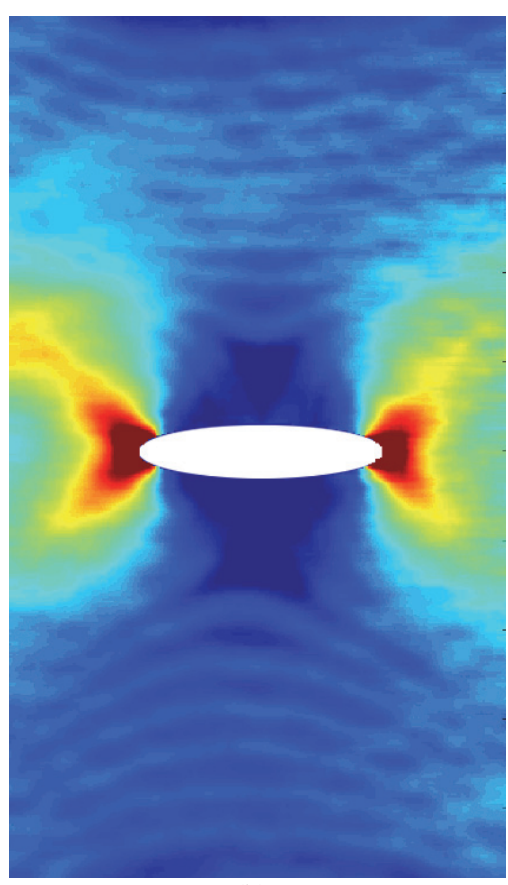

(b)

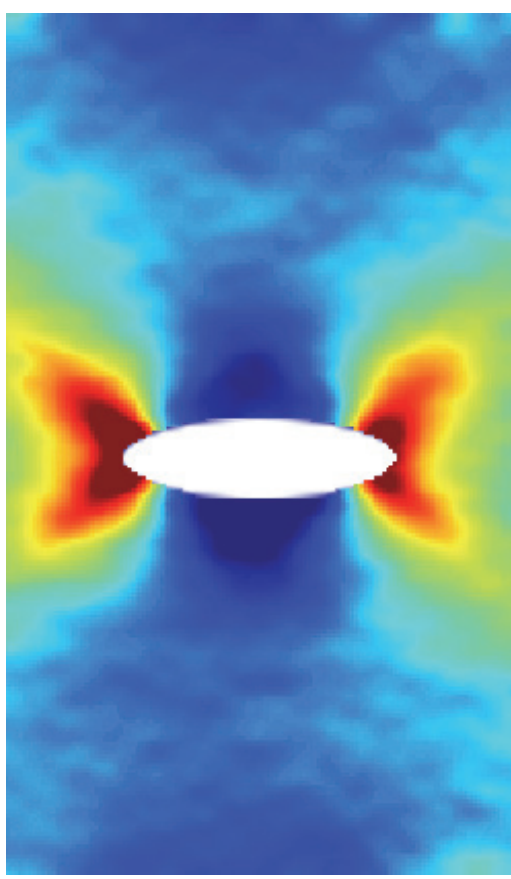

(c)

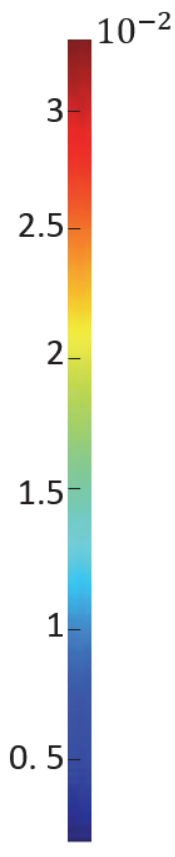

Figure 4: Map of the strain along $\mathrm{X}$ axis [-] of the elliptical hole with an aspect ratio 0.1 normal to the load axis. Results computed, in order of appearance, with (a) LSM, (b) SGM, (c) NCORR.

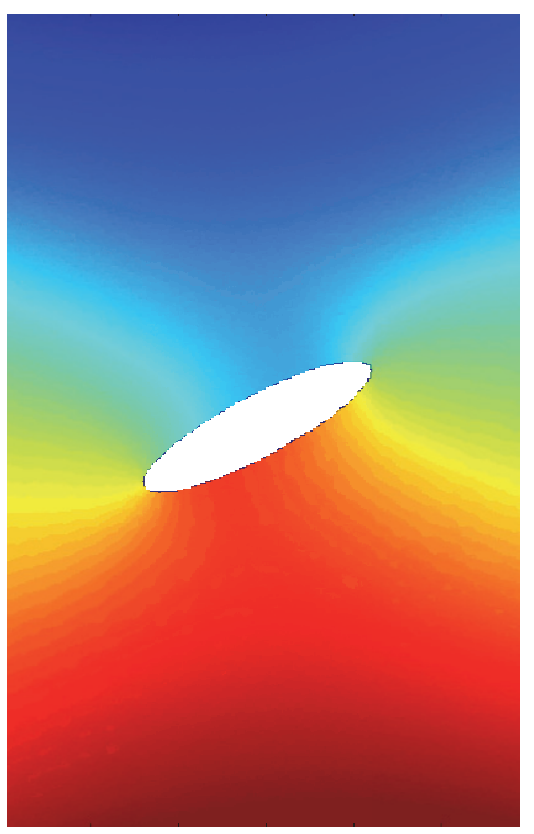

(a)

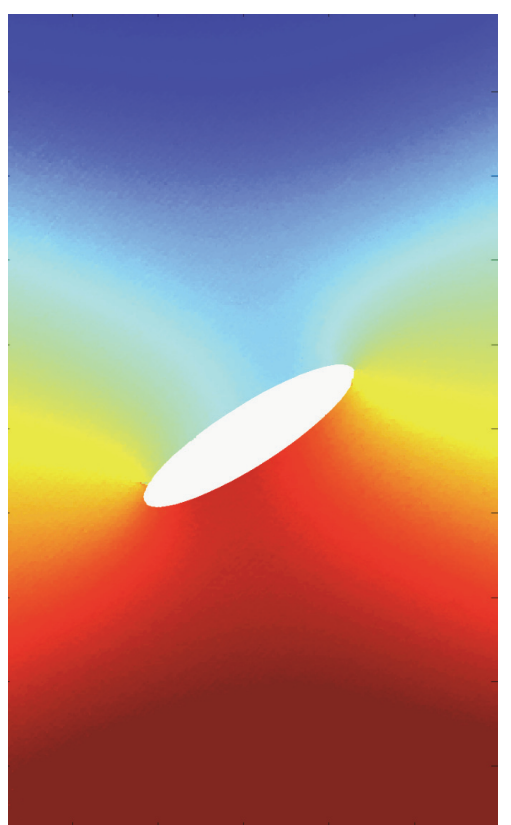

(b)

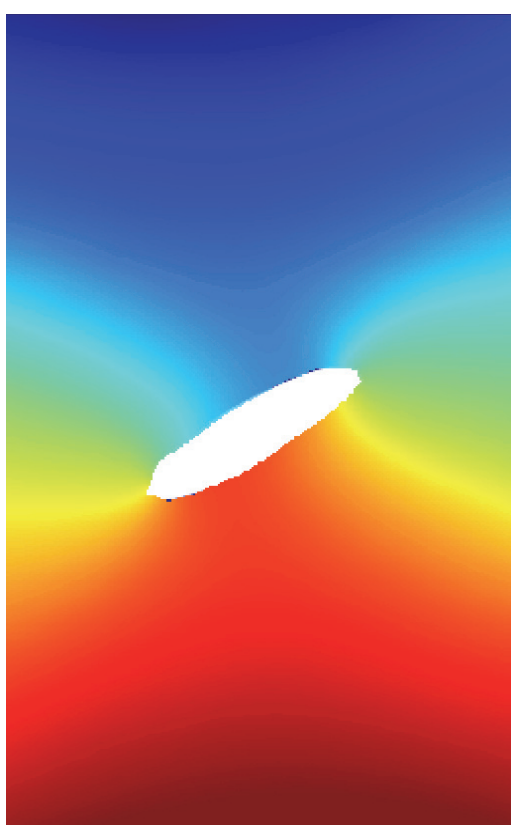

(c)

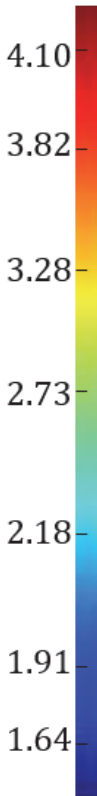

1.64

Figure 5: Map of the displacement along X axis $[\mathrm{mm}]$ of the elliptical hole with an aspect ratio $0.1,60^{\circ}$ inclined with respect to the load axis. Results computed, in order of appearance, with (a) LSM, (b) SGM, (c) NCORR. 


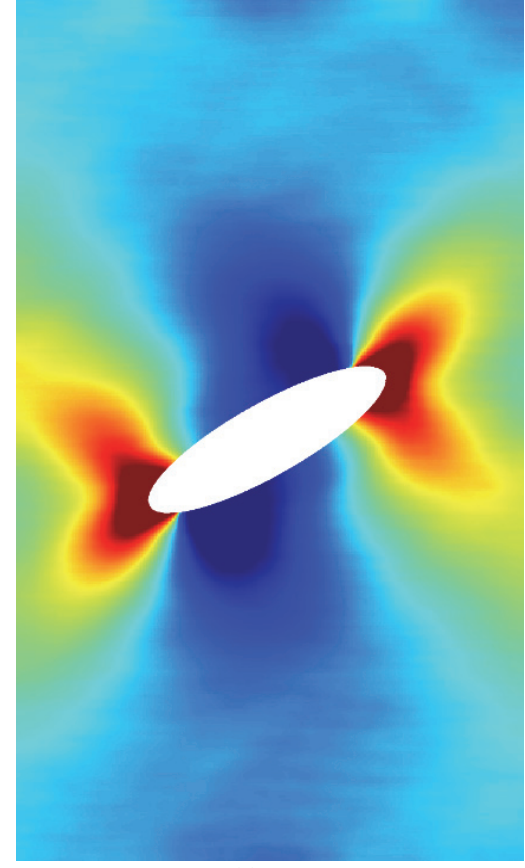

(a)

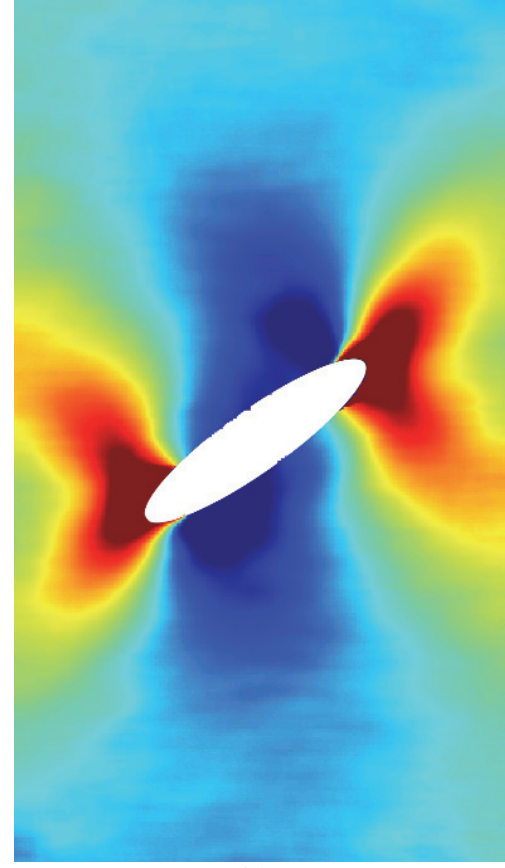

(b)

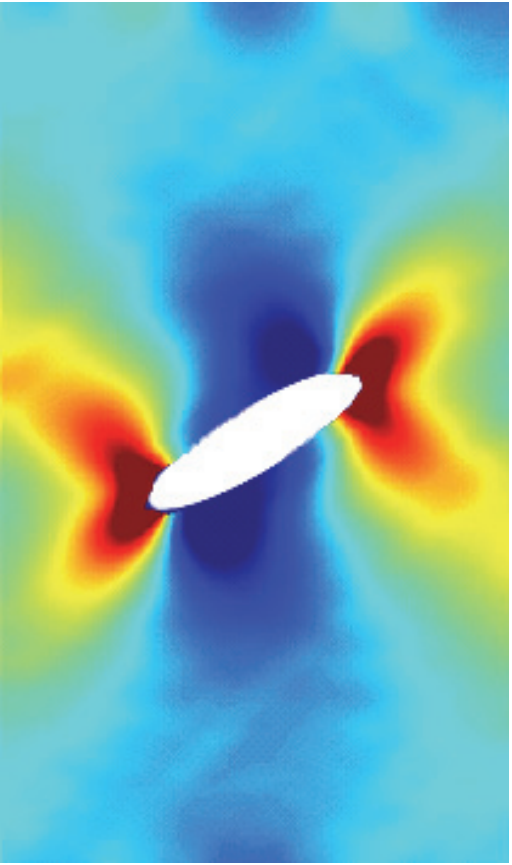

(c)

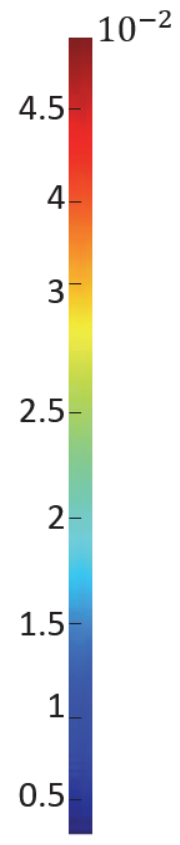

Figure 6: Map of the strain along $\mathrm{X}$ axis[-] of the elliptical hole with an aspect ratio $0.1,60^{\circ}$ inclined with respect to the load axis. Results computed, in order of appearance, with (a) LSM, (b) SGM, (c) NCORR.

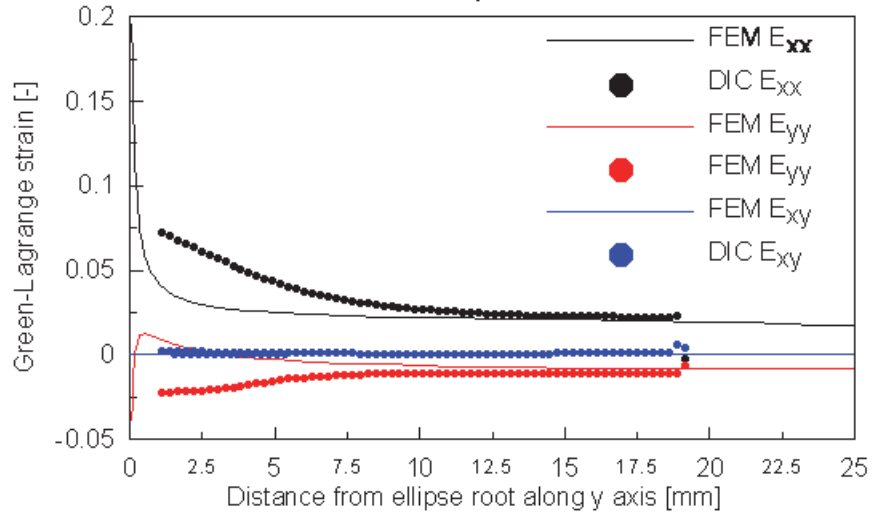

(a)

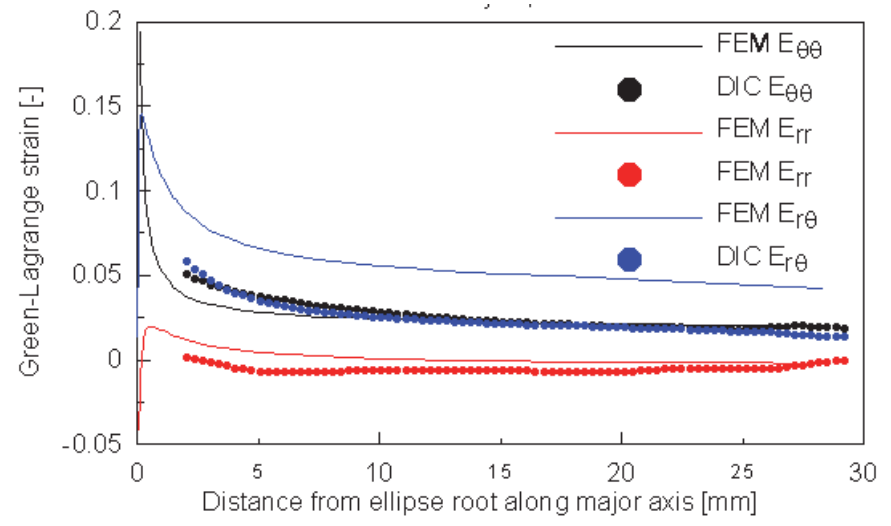

(b)

Figure 7: Green-Lagrange local strain components along the major axis. (a) elliptical hole with an aspect ratio 0.1 normal to the load axis (axial normal strain $=2.01 \%$ ); (b) elliptical hole with an aspect ratio $0.1,60^{\circ}$ inclined with respect to the load axis (axial normal strain $=3.36 \%$.

\section{CONCLUSIONS}

$\mathrm{I}$ $\mathrm{n}$ the present paper, different DIC methods are used to obtain 2D full-field strain maps of plain and notched specimens under tensile loading made of two different highly deformable materials: hot mix asphalt and thermoplastic composites for 3D-printing applications. In the latter specimens, an elliptical hole is introduced to assess the potentiality of the method in experimentally capturing strain gradients in mixed-mode fracture situations. The comparison between the DIC methods being analysed is satisfactory, and the comparison with FEM is good. 
Undoubtedly, as far as high deformable materials are concerned, matching algorithms can experience a lot of different issues: the transformation considered between master and slave image can be inadequate to model localized deformations. Numerical problems, such as pixel-locking, can lead to a misinterpretation of the results. On the other hand taking into account that the displacement solution should present continuity to some extent (even if fractures occur), makes the use of regularization constrain in the matching technique an efficient effort to improve the final results reliability. In this context the use of the new SGM algorithm developed by the authors seems to adequately address such requirement, even if further investigations should be performed to assess its general application in DIC experiments.

\section{REFERENCES}

[1] Grédiac, M., The use of full-field measurement methods in composite material characterization: interest and limitations, Compos Part A: Appl Sci Manuf, 35 (2004) 751-761.

[2] Chu, T.C., Ranson, W.F., Peters, W.H., Sutton, M.A., Applications of digital-image-correlation techniques to experimental mechanics, Exp Mech, 25(3) (1985) 232-245.

[3] Muszynski, L., Wang, F., Shaler, S.M., Short term creep tests on phenol resorcinol formaldehyde (PRF) resin undergoing moisture cement, Wood Fiber Sci, 34(4) (2002) 612-624.

[4] Muszynski, L., Lopez-Anido, R., Shaler, S.M., Image correlation analysis applied to measurement of shear strains in laminated composites, Paper presented at the SEM XI International Congress on Experimental Mechanics. Orlando, FL, (2000).

[5] Choi, S., Shah, S.P., Measurement of deformations on concrete subjected to compression using image correlation, Exp Mech, 37(3) (1997) 307-313.

[6] Kim, Y.R., Wen, H., Fracture energy from indirect tension test, J Assoc Asphalt Paving Technol, 71 (2001) $779-793$.

[7] Seo, Y., Kim, Y.R., Schapery, R., Witczac, M., Bonaquist, R., A study of crack-tip deformation and crack growth in asphalt concrete using fracture mechanics, J Assoc Asphalt Paving Technol, 73 (2004) 697-730.

[8] Masad, E., Muhunthan, B., Shashidhar, N., Harman, T., Aggregate orientation and segregation in asphalt concrete, Proceedings of sessions of geo-congress on application of geotechnical principles in pavement engineering, (1998) 69-80.

[9] Birgisson, B., Montepara, A., Romeo, E., Roque, R., Tebaldi, G., Influence of mixture properties on fracture mechanics in asphalt mixtures, Road Mater Pavement Des, 11 (2010) 68-88. DOI:10.3166/rmpd.11hs.61-88.

[10] Nahshon, K., Hoffman, W. A., Ullagaddi, C. B., In Springer International Publishing, Characterization of Structural Scale Ductile Fracture of Aluminum Panels Using Digital Image Correlation, Fracture, Fatigue, Failure, and Damage Evolution, 5 (2015) 81-87.

[11] Makki, M. M., Chokri, B., Determination of Stress Concentration for Orthotropic and Isotropic Materials Using Digital Image Correlation (DIC). In Multiphysics Modelling and Simulation for Systems Design and Monitoring, Springer International Publishing, (2015) 517-530.

[12] Petrikova, I., Marvalova, B., Samal, S., Cadek, M., Digital Image Correlation as a Measurement Tool for Large Deformations of a Conveyor Belt. In Applied Mechanics and Materials, 732 (2015) 77-80.

[13] Sutton, M. A., Hild, F., Recent Advances and Perspectives in Digital Image Correlation. Experimental Mechanics, 55(1) (2015) 1-8.

[14] Ncorr, Algorithms used in Ncorr, http://www.ncorr.com/index.php/dic-algorithms, (last accessed 15.03.15)

[15] Hirschmuller, H., Stereo Processing by Semiglobal Matching and Mutual Information, IEE Trans. Pattern Analysys and Machine Intelligence, 30(2) (2008) 328-341.

[16] Gruen, A., Adaptive least squares correlation - a powerful image matching technique, in S Afr J Photogrammetry Remote Sens Cartogr, 14(3) (1985) 175-187.

[17] Re, C., Cremonese, G., Dall'Asta, E., Forlani, G., Naletto, G., \& Roncella, R., (2012, November). Performance evaluation of DTM area-based matching reconstruction of Moon and Mars. In SPIE Remote Sensing, (2012) 85370V$85370 \mathrm{~V}$.

[18] Birchfield, S., Tomasi, C., Depth discontinuities by Pixel-to-Pixel Stereo. Proc. Sixth IEEE Int'l Conf. Computer Vision, (1998) 1073-1080.

[19] Kolmogorov, V., Zabih, R., Computing visual correspondence with occlusions using graph cuts, in International Conference for Computer Vision, (2001) 508-515.

[20] Sun, J., Shum, H., Zheng, N., Stereo matching using belief propagation, in IEEE Transactions on Pattern Analysis and Machine Intelligence, 25.7 (2003) 787-800. 
[21] Dall'Asta, E., Roncella, R., A comparison of semiglobal and local dense matching algorithms for surface reconstruction. International Archives of The Photogrammetry, Remote Sensing And Spatial Information Sciences, 40(5) (2014) 187-194.

[22] Pollefeys, M., Koch, R., Van Gool, L., A simple and efficient rectification method for general motion. In Computer Vision, The Proceedings of the Seventh IEEE International Conference, 1 (1999) 496-501.

[23] Westerweel, J., Fundamentals of digital particle image velocimetry. Measurement science and technology, 8(12) (1997) 1379.

[24] Hirschmuller, H., Scharstein, D., Evaluation of stereo matching costs on images with radiometric differences. Pattern Analysis and Machine Intelligence, IEEE Transactions, 31(9) (2009) 1582-1599.

[25] Stein, A. N., Huertas, A., Matthies, L. (2006, May). Attenuating stereo pixel-locking via affine window adaptation. In Robotics and Automation, 2006. ICRA 2006. Proceedings 2006 IEEE International Conference, (2006) 914-921. 